\title{
Membrane processes in nanoparticle production
}

\author{
Pellegrino, J.*;Schulte, L.R. ; De la Cruz, J.; Stoldt, C. \\ *Corresponding author \\ Department of Mechanical Engineering, University of Colorado-Boulder, 1111 \\ Engineering Drive, Boulder, CO 80309
}

\begin{abstract}
With the development of the scanning tunneling microscope, and other high resolution "microscopies", researchers could examineand adapt synthetic process to control particles on the nanometer scale. Since then there have been an explosion of techniques for fabricating nanoparticles. So called "top-down" synthetic techniques start with large particles and make them smaller via grinding, spraying or pyrolysiswhile "bottom-up" techniques start with precursorsand build up to nanoparticles (NPs) using deposition techniques or self-assembly of molecules. Anideal process for fabricating NPs would be tunable to a specific size or shape, usingfew industrially-available, nontoxic reagents, and the produced-NPs should be shelf-stable and in a concentrated suspension, with a narrow size distribution. In this work, we provide a summary meta-review of how membranes have been used to produce $\sim 100 \mathrm{~nm}$ entities and provide observations that membranes could be used to enable rational processesforindustrially-friendly,continuousproduction of NPs. Our meta-review focuses on nano-sized entities created in a suspension versus using the membrane as a potentially "sacrificial" template, thus, we consider three nominal,membrane device operating modes: dispersion, emulsion, and contactor.
\end{abstract}

Keywords: nanoparticle; membrane contactor; reactor, dispersion;emulsion

\footnotetext{
${ }^{1}$ Current address: CMID Inc. 1402 N. Capitol Ave. Suite 250, Indianapolis, IN 46202
} 


\section{Introduction}

Nanoparticles (NPs) are of interest becausethey adhere to quantum rather than classical Newtonian mechanics. Because of this, materials at the nanoscale have different electrical, thermal, magnetic, optical, and mechanical properties than in the bulk. NPs are defined in several different ways(depending on the scientific community). Most agree, that to be classified as a NP, at least one dimension is between 1and 100nm. The US Patent and Trademarks Office (USPTO) has an additional requirement that a nanostructure have some property, function or effect that is attributed to the size of the structure.Some scientific communities deviate from these definitions, perhaps most notably, the medical community uses NP to encompass materials that are several hundred nanometers in size.

NPs have been used for several millennium by people all over the worldhistorically, first by the Greeks to color hair black, then to color red pottery and art in China and windows in a variety of places in the European continent[1, 2]. In 1974, Taniguchi first coined the term "nanotechnology" and in 1981, with the development of the scanning tunneling microscope (STM),researchers could examine and manipulate individual atoms[3].

NPs are playing a major role in future technologies. In medicine, encapsulation and particulate formulations can finetune the bioavailability and solubility of the drug in the body and improve therapeutic effects or decrease unwanted side effects[4]. Outside of medicine, nano-sized materials are being studied for a variety of applications includingcatalysts, electronics, ceramics, sensors, pigments, and in spectroscopy[5-7].

Nanotech companies have existed since at least 1997 with the startup Zyvex Technologies. Commercially, NPs are being exploited for their unique properties, as mentionedabove. NPs are being sold by a variety of companies, one of the largest of which is OCSiAl, which produces and sells nanotubes as an additive to enhance the mechanical strength, and electrical and thermal conductivity of materials.In 2013, the company started a plant, which could produce up to 10 tons of single walled carbon nanotubes,priced at several thousand dollars per $\mathrm{kg}$. While commercially available, these production numbers are still small compared to potential end-use opportunities. 
There are many methods of producing nanosized particles and structures. Some techniques start with large particles and use comminution techniques (top-down manufacturing), while others build upthe NPs, using deposition techniques or selfassembly of molecules (bottom-up manufacturing). There are a variety of ways to synthesize nanosized-films such as, atomic or molecular layer deposition (ALD and MLD)[8], Langmuir-Blodgett-type[9] — which uses the hydrophilic and hydrophobic moieties of amphiphilic molecules to arrange themselves into a pattern - and lithography, which uses a variety of electro-magnetic fields (often $200 \mathrm{~nm}$ UV radiation)to imprint a pattern onto another material[10].While by no means specific to the creation of NPs, these examples illustrate that there are many ways to manipulate materials on a nanoscale.

Notwithstanding their importance, and that there are companies selling useful NPs, they are still expensive, and it is difficult to produce large quantities of product to precise specifications. An ideal process would be tunable for producing a variety of nominal sizes, with a narrow size distribution. The process should be scalable and use non-toxic reagents. The final product should be a concentrate with little or no traces of reagents and be shelf stable for at least months.It is by considering these aspects of production wherein membranes could be especially useful.

Membranes are attractive for production of NPs for several reasons. Foremost of which is thattmembrane devices are easily scalable, for example, to produce more droplets in the dispersion and emulsion case, or to have more reaction surface area in the contactor case, another membrane module can be added to an array. Membranes are commercially available on an industrial scale as both polymers and ceramics, and thus have proven their compatibility with the range of temperatures, pressures, and chemical environments that are required for production of the wide variety of nano-sized materials. Membranes are already proven-technology in a variety of industries at a large commercial scale[11], e.g., dialysis in medicine, metal ion recovery in the mining industry, whey concentration in dairy applications, and water production by reverse osmosis, to name a few. We suggest that membranes may be studied in a more systematic fashion for NP production because they represent a unique opportunity for scalable nanomanufacturing of an emerging class of high-value products. 
We must note that there other clever approaches being developed to scale up the throughput and robustness of NP synthesis $[12,13]$, especially based on microfluidic (microreactor) platforms[14-17]. Interestingly, and illustrative of the fast-adoption of multiple (three in this case) innovations, Riche et al. [18] described a "parallelizable", high-throughput method for creating Pt NPs in an ionic liquid, using 3-d printing to create their "device". Nonetheless, clear process-oriented comparisons between microfluidic and membrane-based approaches for making NPs have not been systematically pursued. For example, Jahn et al. [19] provided a comparison of lipidbased vesicle particle size distribution obtained with membrane extrusion versus a microfluidic device, with the latter being narrower, but no details on the system was provided. Thus, at present it is difficult to make techno-economic comparisons that would really address the most optimal approaches for NP scale-up.

The purpose of this review and commentary is to extend upon, and focus more consideration onto, the role that membrane reactors can have in creating beneficial NPs for diverse industrial applications. We highlight literature examples in which nano-sized entities (generally $\leq 100 \mathrm{~nm}$ but, at least, $<1 \mu \mathrm{m}$ ) have been created. Clearly, membrane processes can be used to produce NPs in a variety of ways. In the schematic illustrations inFigure 1, we identify the three modes we have chosen for binning our literature review's examples and discussion. These are dispersion, emulsion and contactor (or interfacial, self-assembly) approaches.

With the dispersion case, usually a micro- or ultrafiltration membrane (MF or UF), having pores on the order of $10 \mathrm{~nm}$ to 10 microns, is used to produce and inject precursor droplets into the bulk phase. These can then react in the bulk phase with another species to produce the NP. In the emulsion case, the droplet injected through the membrane (into some medium) is the "particle" produced by the membrane process. In our binning, this emulsion approach can include crystallization or phase separation due to a miscibility gap between a component in the droplet permeated through the membrane and the continuous medium it enters. In the contactor or interfacial, self-assembly case, the membrane acts as a phase contactor with a reaction taking place on or near the surface, i.e., at the pores of the membrane. We consider this to be a process in which 
there is insignificant pressure-driven flux through the membrane, ideally only diffusive mass transfer.
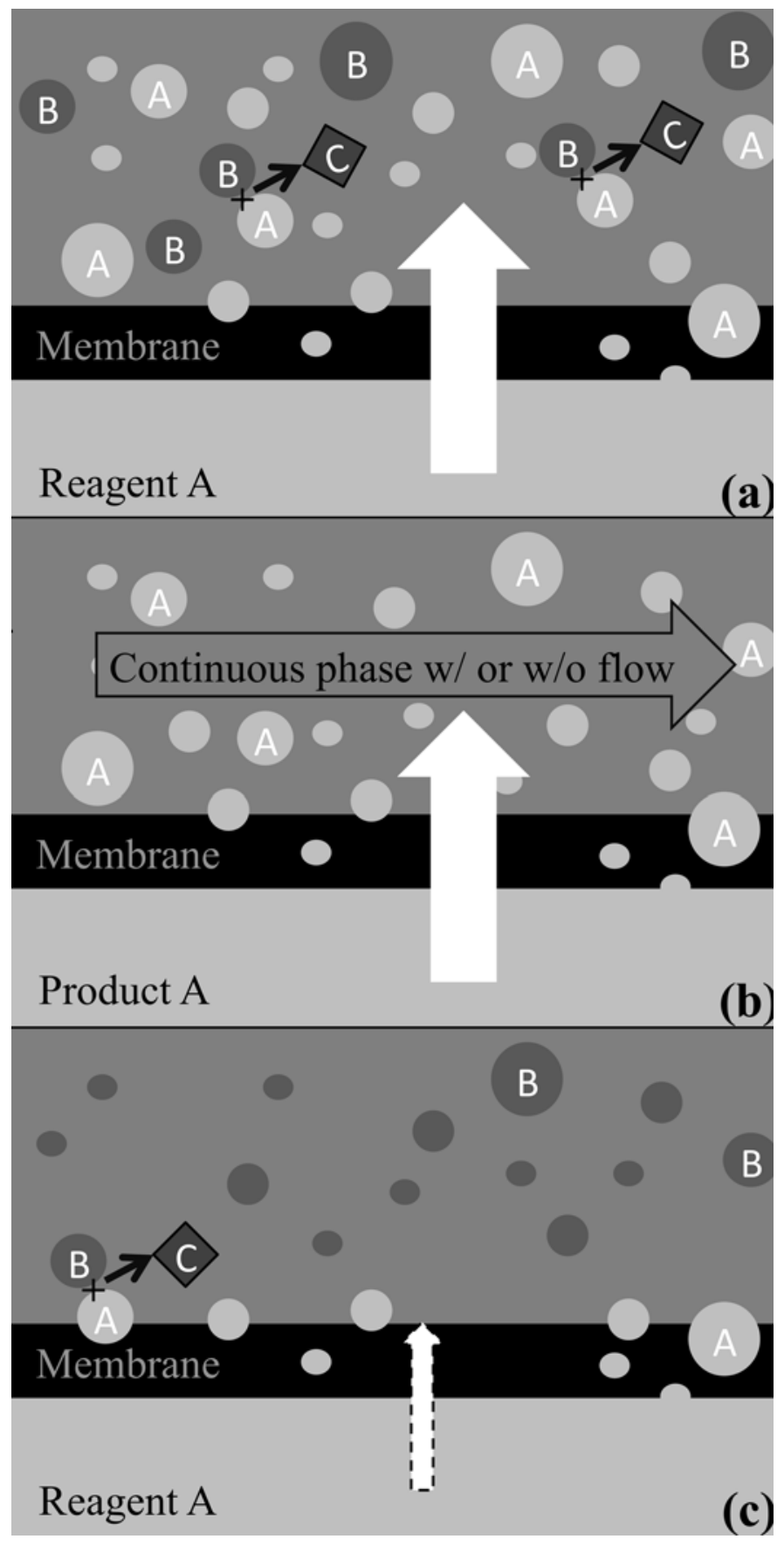

Figure 1. Membranes can be used in several ways to manufacture NPs. (a) In dispersion mode, reagent $\mathrm{A}$ permeates across the membrane and mixes and reacts with reagent $\mathrm{B}$ in a bulk medium to form product $\mathrm{C}$; (b) in emulsion mode, the product $\mathrm{A}$ reaches desirable dimensions as a dispersion in a continuous phase by passing through a membrane - a phase change or precipitation may also occur, but not a chemical reaction, and (c) in contactor mode, reagent $\mathrm{A}$ forms a membrane-stabilized interface with reagent $\mathrm{B}$ to form product $\mathrm{C}$ by reactioninsignificant convective flux of $\mathrm{A}$ is applied. 
It is important to note that the latter category, the membrane contactor, could be expanded to include "sacrificial" or "immobilization" processes. For example, wherein a nanomaterial is created within a membrane, which acts as the template $[20,21]$. Additionally, groups have used in-situ formation of NPs on or within the pore structure of membranes to facilitate a type of reactive membrane, e. g., Smuleac et al., [22, 23] described synthesis of $\mathrm{Fe}$ or $\mathrm{Fe} / \mathrm{Pd}$ nanoparticles within a chemically-modified microfiltration membrane, which could then perform oxidation of organic contaminants in water.Also, there is the in-situ creation of nanoparticles to create a membrane with novel properties, such as, nano- $\mathrm{TiO}_{2}$ domains within a poly(amide-imide) membrane [24]. While these approaches are certainly useful and interesting for specific end-uses, this review focuses on examples wherein the membrane could facilitate a continuous or fed-batch process configuration.

There have been several prior reviews of the general subjects of membrane dispersion reactors[25-27] and membrane emulsification[28-34] processes which provide the overarching context and important background and perspectives for the current work. Also, reviews on the subject of membrane crystallization [35]definitelyare relevant tomembrane process development for production of nano-sized entities. Thus, herein we are presenting something of a meta-reviewfocusing on the prospects for membrane processes as scalable nanomanufacturing platforms for NPs. We initially outline the general features, mechanisms, and membrane material and geometry aspects for the membrane processes involved. We then list, and discuss simply, examples that fit within our targeted criteria, and conclude with final perspectives and commentary.

\section{Membrane-based particles and droplets}

A membrane serves to stabilize an interface, with constant surface area, across which mass (and heat) transfer (and maybe a reaction) can occur. In principle, the mass transfer across that interface may be predominantly diffusive or convective, or a combination of both, depending upon the transmembrane driving forces applied. Most frequently, a porous membrane (nominal mean pore size $10 \mathrm{~nm}$ to $10 \mu \mathrm{m}$ )can be used to introduce an immiscible dispersed phase into a bulk (or continuous phase) as droplets, using a mechanical pressure gradient.Also, a second miscible phase may also be added 
through the membrane, thus, the membrane then acts as a very effective micromixer to reduce the length scales for subsequent diffusion of reactants into the bulk phase. The following considers these general features in some more detail.

\subsection{Droplet size}

Early experiments and analysis[36], and subsequent numerical modeling (e.g.,[33, 37]), have provided the mechanistic viewpoint that as a droplet emerges from a surface poreit likely has a neck that connects it to the pore (seeFigure 2). The amount of fluid in the droplet + neck before it "pinches off" governs the final diameter of the separated droplet.

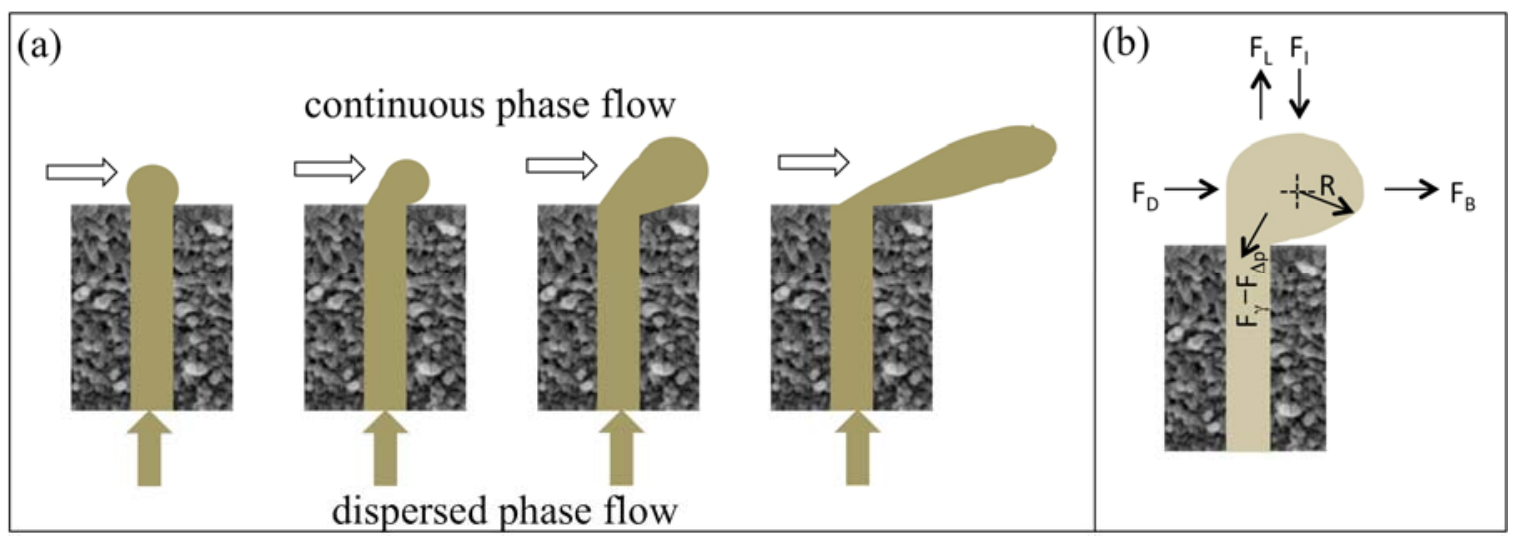

Figure 2. (a) The evolution with time of a droplet + neck (of the dispersed phase) emerging from a surface pore (adapted from [33]. (b) The general force balance on an emerging droplet + neck (with nominal radius $\mathrm{R}$ ), wherein $\mathrm{F}_{\mathrm{D}}=$ drag force; $\mathrm{F}_{\mathrm{L}}=$ dynamic lift force; $\mathrm{F}_{\mathrm{I}}=$ inertial force; $\mathrm{F}_{\mathrm{B}}=$ bouyancy force; $F_{\gamma}=$ interfacial force; $F_{\Delta p}=$ static pressure difference force (adapted from [31]).

The evolution of this final droplet/neck shape and size will be controlledby a dynamic force balance that includes:

- disjoining pressure of the droplet fluid that retards its self-separation;

- the porous network that exists below the surface pores can also influence whether or not internal "pinch-off" of an emerging droplet occurs before a neck evolves at the membrane surface;

- the local pressure difference and the interfacial energy between the continuous and dispersed phases that governs the Laplace relationship between thelocal curvature and the internal pressure within a droplet;

- local shear stresses and bouyancy forces acting on the droplet/neck shape; 
- wetting (or not) of the membrane surface by the growing droplet/neck shape; and

- interference between droplets growing from multiple surface pores proximal to each other.

Notwithstanding the complexity of any real scenario, the model problem has lent itself to a general scaling relationship presented in Boom and Schroen [33] as

$$
\frac{d_{d}}{d_{p}} \geq \sqrt{\frac{2 \gamma}{5.1 \tau_{w} d_{p}}}=\sqrt{\frac{1}{5.1 C a}},
$$

where $d_{d}$ and $d_{p}$ are the nominal droplet and pore diameters, respectively; $\tau_{w}$ is the shear stress at the wall; $\gamma$ is the interfacial tension; $C a=(\eta v / \gamma)$ is the Capillary number; $v$ is the velocity at the droplet half height; and $\eta$ is the shear viscosity. This correlation should only be used as a general guide as an oft-stated (e.g.,[38]) heuristic is that the droplets formed are 2-5 times the size of the membrane's nominal pore size. The broad range of the results for $d_{d} / d_{p}$ is likely due to the previously listed items in the general force balance, and the fact that the system is dynamic and not at equilibrium, especially with respect to the interfacial tension in any multicomponent mixture.Gijsbertsen-Abrahamse et al.,[30] provided a tabulated (qualitative) assessment of the influence of the various parameters on the droplets' figures-of-merit: mean diameter, size dispersity, and productivity (flux); and Charcosset et al., [31] tabulated some further quantitative values.

If NPsare being formed by a reaction between a specie(s) in the emerging droplet and the continuous phase, it is also reasonable to presume that nanocrystals may be nucleating, growing, aggregating, and dispersing from this drop continuously. Thus, it couldbe an even richer challenge to assess the physics-based scaling rules between process variables and NP mean size and distribution.

The influence of process conditions and membrane material properties on droplet size, polydispersity, and productivity (flux) gleaned from studies of membrane emulsification will be quite applicable to the production of NPs using the modes of dispersion and emulsification discussed previously (Figure 1).The simplest relation [33] between pore size and particle size, as mentioned above, is the linear oned $d_{d}=c d_{p}($ with values of $c$ typically between 2 and 5).Also, the minimum pressure required to push the dispersed phase though the membrane, producingan emulsion, can be estimated from the 
Laplace relation $\Delta p_{c}=4 \gamma / d_{p}$. Thus, to produce NPs from smallmean pore diameter membranes, using the dispersion or emulsion modes, the required transmembrane pressures can become quite high [39].

For illustrative purposes, the pressure required can be estimated for extremes in interfacial tension and pore size. Interfacial tension is usually between 1 and $50 \mathrm{mN} / \mathrm{m}$, with $1 \mathrm{mN} / \mathrm{m}$ representing two polar liquids, and $50 \mathrm{mN} / \mathrm{m}$ representing a non-polar and a polar liquid [40]. Track-etch membranes, which have uniform pore sizes as low as $15 \mathrm{~nm}$ and low porosity - thus minimizing pores interacting with each other - are attractive for producing emulsions of small and uniform size. Furthermore, Shirasu porous glass (SPG) membranes, which are one of the the most studied membranes in emulsification, can have pore sizes in the $45-85 \mathrm{~nm}$ range [34]. If the $d_{d} / d_{p}$ is assumed to be 2 , the best-case scenario for producing small particles, and physically possible as noted by the literature, we can estimate the critical pressures required to make particles from 30 to $100 \mathrm{~nm}$ in size, using membranes with 15 and $50 \mathrm{~nm}$ pores. The smallest pore sizes require a $\Delta p_{c}$ of 130 bar, and thus will present serious technical challenges; however, using membranes with $50 \mathrm{~nm}$ pore sizes is more feasible, as it requires $\Delta p_{c}<40$ bar. These estimates ignore potentially important effects of the composition of the dispersed and continuous phase, and the viscosity of the continuous phase and the dynamic interfacial properties of the emulsifier, which are known to affect size at operating pressures just above the $\Delta p_{c}$. Nonetheless, on a first "look", it appears plausible that smaller sized emulsions could be fabricated by purposeful choice of membrane and operating parameters.

But such high pressure conditions may be less pertinent for the mode of contactor.Figure 3 (middle) illustrates a porous $\alpha$-alumina UF membrane (bottom end sealed) whose lumen was filled with a solution of $\mathrm{AgNO}_{3}$ in water, being dipped into a solution of heptanoic acid and tetra butyl ammonium borohydridein toluene. Silver (Ag) NPs can be seen "streaming" from the surface of the membrane in response to a gentle rotation of a stirrer bar at the base of the vessel. No mechanical transmembrane pressure gradient has been applied. Our group is actively investigating the mechanisms underlying such NP growth. 

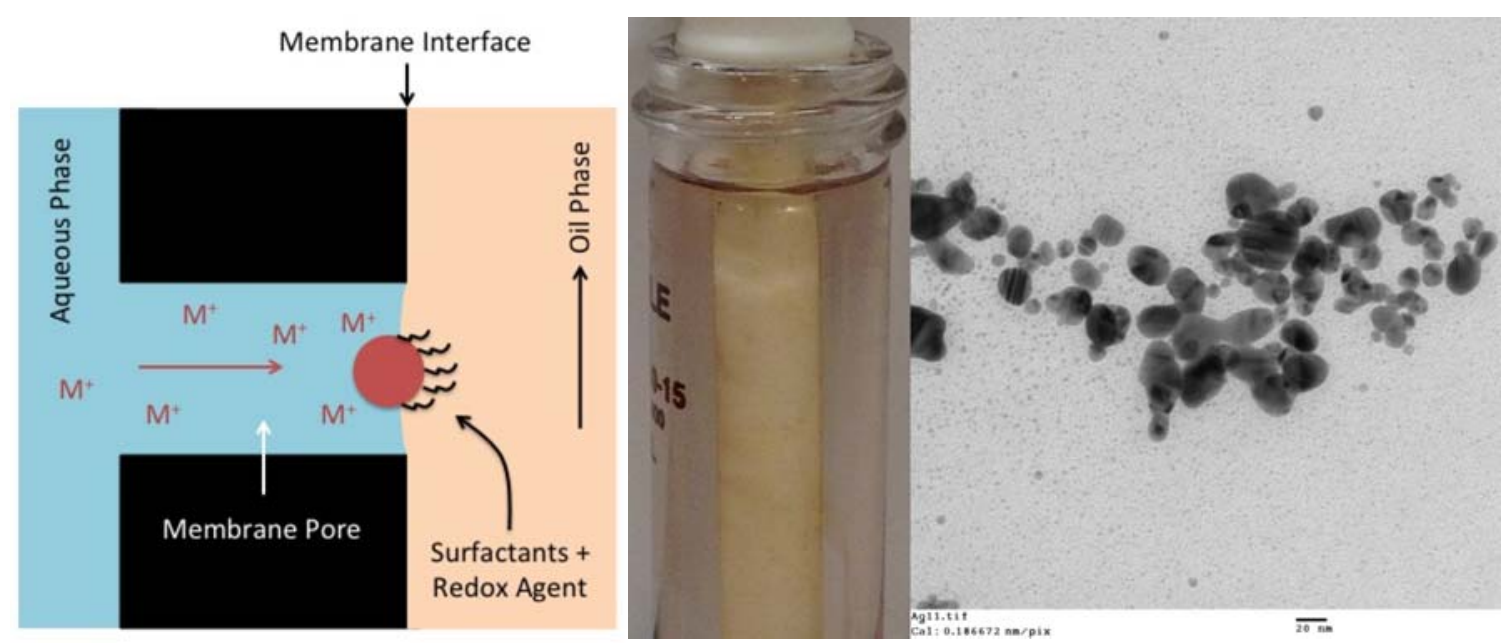

Figure 3. (left) An illustration showing hypothetical metallic nanocrystal's formation at a pore's oil-water boundary in a membrane contactor reactor; and (middle) photo taken during the synthesis of Ag nanocrystals using this membrane contactor approach - the pale ceramic membrane, containing an aqueous solution of $\mathrm{Ag}^{+}$, is submerged vertically into the reaction vessel. The reaction vessel contains a mixture of organic solvent, a long-chain carboxylic acid surfactant, and a reducing agent. Ag nanocrystals, appearing reddish-pink in the photo, are observed to nucleate and grow across the membrane, and subsequent mixing releases them into the solution-(right) TEM image showing the Ag nanocrystal product, with size below about 40 nm.

\subsection{Membranes}

The range of membranes applied to making droplets and particles has been quite broad (e.g., see the tables in [30, 31]. A favorite for membrane emulsification has been the Shirasu Porous Glass (SPG) tubular membranes made as a phase separated, acidleached porous glass of $\mathrm{Al}_{2} \mathrm{O} 3-\mathrm{SiO}_{2}$ skeleton, with nominal mean pore size and porosity of 0.05-20 $\mu \mathrm{m}$ and 50-60\%, respectively. Further description and specialized references may be found in Vladisavljevic et al. [41].Membrane modules based on microporous hollow fibers fabricated from polypropylene $[42,43]$ and polyethersulphone $[42,44]$ have also been used, and they represent an attractive pathway for industrial applications.

Highly uniform idealized membranes, such as track-etched polycarbonate[45, 46], anodic alumina[45], and, lithographically-produced microsieve membranes[39, 47] have also been used for both theoretical and demonstration purposes. It is important to note that the studies of Boom and colleagues [48] suggest that surface porosity should be quite low $(\sim 1.5 \%)$ in order to minimize the overlap of dispersed droplets, which probably leads to increased polydispersity.We indicate and comment on more membranes in the following specific sections. 


\subsection{General modeling}

Beyond the modeling initiatives on droplet formation previously mentioned, Gijsbertsen-Abrahamse et al., [30]provided algebraic scaling relationships to estimate thecritical pressure to disperse a drop; the membrane resistance; and the membrane area required as rational starting points for industrial cross-flow membrane applications.

From the research perspective, there have been several modeling efforts reported that were suitable to specific experimental benchmarks. Kieffer et al., [42] created a numerical multiphysics model which solved the axi-symmetric, momentum (NavierStokes) and mass transfer (advection and diffusion) with reaction (the crystallization) conservation equations to predict the degree of supersaturation in their experimental modules. The numerical results were color-coded and compared to experimental $\mathrm{x}$-ray density maps of the crystal density in the modules and reasonable agreement was obtained.

Li et al., [49]performed microscale modeling to predict supersaturation as a function of time in a diffusive mass transfer environment between a dispersed droplet and the continuous phase. They included a population (size) balance model that has nucleation, growth, and aggregation, and uses the supersaturation index from the mass balance to predict the particle density evolution versus time. Good agreement was obtained with their small scale, membrane dispersion reactor'sphysical results.

Jia and coworkers $[43,50,51]$ focused their modeling of gas-liquid contactors to forecasting the gas absorption rate. A theoretical resistances-in-series model including the liquid phase equilibrium (and reaction), the membrane's mass transfer resistance, and the module's boundary layer mass transfer was used to forecast the absorption rate in their experiments. This was then compared to the measured values of gas uptake (by mass balance in the experiments) and reasonable agreement was obtained in some situations but less so in others. This is commented on further in a later section.

\section{NP synthesis via dispersion mode}

A summary of the nano-sized particles that have been synthesized using a membrane to disperse one species into a continuous phase are listed inTable 1.Jia and $\mathrm{Liu}[27]$ have already provided a thorough review of liquid dispersion processes 
producing NPs using a membrane. They concluded that the use of a membrane to disperse one phase into another results in narrowed size distributions of NPs due to the more uniform concentration of reactants near the membrane surface from enhanced micro-mixing on the nano and micro-scale. This section will add to their review by focusing on the gas-liquid dispersions via membranes.The droplets, whether an inert gas or one of the reagents, mayalso serve to enhance mixing.

Table 1. NPs synthesized using membranes in dispersion mode.

\begin{tabular}{lllll}
\hline Membrane material & Pore size $(\mu \mathrm{m})$ & $\mathrm{NP}$ composition & Particle size $(\mathrm{nm})$ & Ref. \\
\hline PS, PDC, PES & -1 & $\mathrm{BaSO}_{4}, \mathrm{CaCO}_{3}$ & $15,30-60$ & {$[52]$} \\
PS, PDC, PES & -1 & $\mathrm{BaSO}_{4}$ & 70 & {$[53]$} \\
$\mathrm{Ni}$ & 0.2 & $\mathrm{TiO}_{2}$ & $10-20$ & {$[54]$} \\
$\mathrm{Ni}$ & 0.2 & $\mathrm{SiO}_{2}$ & $10-150$ & {$[55]$} \\
$\mathrm{Ni}, \mathrm{SS}$ & $0.2,5$ & $\mathrm{CaHPO}_{4}$ & $200-600$ & {$[56]$} \\
$\mathrm{Ni}$ & $5,10,20$ & chitosan-TPP & $90-100$ & {$[57]$} \\
Unknown & 0.5 & $\mathrm{BaSO}_{4}$ & $40-200$ & {$[58]$} \\
SS & $1,5,20,50$ & $\mathrm{ZnO}_{3}$ & $7-35$ & {$[59]$} \\
SS & 5 & $\mathrm{BaSO}_{4}$ & - & {$[60]$} \\
SS & 5 & $\mathrm{BaSO}_{4}$ & $20-200$ & {$[61]$} \\
SS & 5 & $\mathrm{FePO}_{4}$ & $40-60$ & {$[40]$} \\
SS & 5 & $\mathrm{Li}_{2} \mathrm{CO}_{3}$ & $30-200$ & {$[62]$} \\
SS & 5 & $\mathrm{pseudoboehmite}$ & $5-20$ & {$[63]$} \\
SS & 5 & $\mathrm{SiO}_{2}$ & $10-25$ & {$[64]$} \\
SS & 5 & $\mathrm{ZnO}_{3}$ & 10 & {$[65]$} \\
SS (tube-in-tube) & $5,10,20,40$ & $\mathrm{BaSO}_{4}$ & $30-150$ & {$[66]$} \\
SS & $5,10,30,50,90$ & $\mathrm{CaCO}_{3}$ & $34-110$ & {$[67]$} \\
Glass & 20 & $\mathrm{CaCO}_{3}$ & $200-400$ & {$[68]$} \\
Unknown & 200 & $\mathrm{CaCO}_{3}$ & 30 & {$[69]$} \\
SS & 600 & $\mathrm{SiO}_{2}$ & $10-30$ & {$[70]$} \\
\hline SS & 5 & &
\end{tabular}

$\mathrm{SS}=$ stainless steel, $\mathrm{PS}=$ polysulfone, $\mathrm{PDC}=$ polydioxanone, $\mathrm{PES}=$ polyethersulfone ${ }^{1} \mathrm{UF}$ membranes with a $\mathrm{MWCO}=1 \mathrm{~K}, 3 \mathrm{~K}, 10 \mathrm{~K}, 30 \mathrm{~K}$

\subsection{Membrane materials}

Of the literature reviewed (see Table 1), metallic membranes dominated the NP dispersion landscape, with nickel and stainless steel being the most popular. Nonetheless, there is no reason to exclude the use of less expensive polymeric materials, as many of the reactions were not conducted under high temperatures and/or aggressive chemical environments.For example, the highest synthesis temperature found in the literature was $90^{\circ} \mathrm{C}$ but most were performed at close to room temperature[69]. The chemical environments of these reactions were similarly benign. Many types and classes of 
reagents, especially gases, such as $\mathrm{CO}_{2}$ and $\mathrm{N}_{2}$, which are also commonly processed by the membrane industry, were utilized[58, 59, 62, 63, 68, 69].

It is important to note, that in some NP synthetic reactions, it is also necessary to consider the electrochemical potentials of any ionizable species (for example, when synthesizing magnetite [71]), in order to mitigate possible undesired redox or ion exchange reactions with the membrane. Moving away from metallic membranes - to their ceramic and polymeric counterparts - might be essential to expanding the types of NP synthetic chemistries accessible for membranes and in lowering costs so that progression from lab to industry-scale is more economically feasible.

\subsection{Membrane nominal pore size}

As stated previously, there appears to be no absolute relationship between the choice of nominal membrane pore size and NP size. A $600 \mu \mathrm{m}$ pore size can make 10-30 nm particles just as a $0.2 \mu \mathrm{m}$ pore size membrane can[70]. Furthermore, we see in Table 1 that nominally $5 \mu \mathrm{m}$ porescreated particles between 5 to $200 \mathrm{~nm}$. Tunable particle size appears controlled by the "degree of mixing" and, while "membrane-less" applications such as rotating packed beds, stirrers, and jets can also produce eddies on the order of 10 $100 \mu \mathrm{m}$, with further mass transfer occurring only by diffusion, a characteristic of membranes is that they can lower the characteristic diffusion length even further[53]. Mixing is not just controlled by pore size, but faster flow rates of either the dispersed or continuous phase can improve mixing [67]. Some of the cited-research used "membranes" which have pore sizes that are larger even than microfiltration (MF) membranes - the latter typically defined as having a pore size from $100 \mathrm{~nm}$ to $10 \mu \mathrm{m}$. These papers were included because the mechanism for delivering the reagents in the larger-pore size membranesmirror their smaller-pore sized counterparts.

\subsection{Use of stabilizer and surfactants}

Several authors have investigated using stabilizer and surfactants as methods to influence NP quality and size.. The use of surfactants is common in NP chemistry but not always present in dispersion membrane systems[72]. Jiang et al.[68] produced nanocalcium carbonate particles as small as $200 \mathrm{~nm}$ in diameter by dispersing $\mathrm{CO}_{2}$ gas through a glass membrane with $20 \mu \mathrm{m}$ pores into an aqueous $\mathrm{Ca}(\mathrm{OH})_{2}$ slurry stabilized 
with oleic acid. It was concluded that oleic acid served two contradictory roles; during the reaction, it caps growth, butafter the reaction its $\pi$-bond interactions increases aggregation of some particles. Other studies $[64,69]$ included a variety of surfactants, such as, carboxymethylcellulose, polyethylene glycol, and sodium stearate.

\section{4. $\quad$ Modeling}

Some models for describing the crystallization process have been presented.Li et al.,[49] assumed that precipitation occurs in a spherical boundary layer surrounding the dispersed phase. Precipitation occurs due to supersaturation and the material balance gives rise to a particle number density distribution within an overarching population balancerelationship [73]. The thickness of the boundary layer is the primary adjustable fitting parameter. Du et al.,[58]used the Villermaux/Dushman method[74] to characterize their micromixing with and without the introduction of bubbles. Then, using the same modeling framework and population balance method as Li et al., [49], with an adjustable parameter for the mixing length, they could reasonably predict size and size distribution of NPs.Near perfect mixing could be achieved by mixing one reagent with finely dispersed $\mathrm{N}_{2}$ bubbles, and then dispersing the second reagent into the first $\mathrm{N}_{2}$-mixedreagent.

\section{NP synthesis via emulsion mode}

Typically, an emulsion has an immiscible fluid dispersed as droplets in another liquid phase, often enabled by surfactant addition. The most common emulsions are oilin-water $(\mathrm{O} / \mathrm{W})$ or water-in-oil $(\mathrm{W} / \mathrm{O})$. For an introduction to emulsions, readers are guided to several review papers on the production of uniform droplets [41] and membrane emulsification that have been previously published[30, 32, 34]. This review focuses primarilyon particles in the sub-900nm range. It should be noted that sub-micron

emulsions have been fabricated using non-membrane technologies since at least the late 1980s and by membrane emulsification since at least the early 1990s[75, 76].Vladisavljevic et al. [77] haveprovided an interesting comparative study between two types of inorganic membranes used as membrane emulsifiers; a high energy (pressure) microfluidizer; and a Si-based microchannel, to perform emulsification of rapeseed oil into surfactant containing water. In these experiments, sub-micron sized NPs were only 
produced with the high-energy microfluidizer, but significantly tighter particle size distribitions were obtained with the SPG membrane. A subsequent review [41] covers these processes (and other microfluidic approaches to producing uniform droplets) in much greater detail.

We have also appropriated othernano-entity synthesis processes under our mode/category of "emulsion". We have included all the processes that permeated a mixture through a membrane but did not incorporate a reaction during the nano-entity's synthesis. We understand that this may be a source of contention. Nonetheless, we chose this to stimulate imaginative thinking, but to avoid too many binnings in this review-not to minimize the process innovations extant using various physiochemical transformations.

Following this rubric, only a few researchers have prepared emulsions that meet the strict definition of a NP-having a dimension less than 100nm. Nonetheless, we have included several studies that have prepared emulsions with an average size of a few hundred nanometers(Table 2) in order to approach the NP domain. While the most common size might be larger than $100 \mathrm{~nm}$, producing emulsions with a membrane results in emulsions with a distribution of sizes. The smallest droplets in these distributions are sub-100 nm in size, proving it is possible to produce nano-sized entities with themembrane emulsification mode.

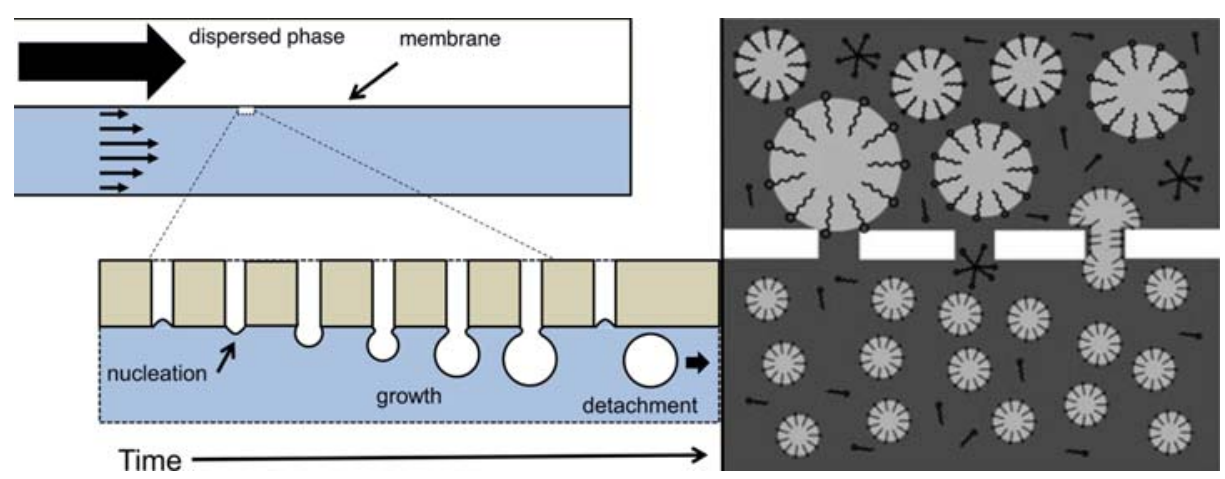

Figure 4. Schematic of (left) crossflow emulsification (possible surfactants omitted) and (right) premix emulsification (from [78])

There are twooft-reported methodsfor emulsion production using a membrane, crossflow emulsification and premix emulsification[41] (see Figure 4). In crossflow emulsification, the to-be-dispersed phase is passed through the membrane and then advected off the surface by the flowing continuous phase. In premix emulsification, a 
coarse dispersed (emulsion or pre-emulsion) phase is already in the continuous phase, and is pushed through the membrane to reduce the droplet size and particle size distribution (PSD). These two processes can be combined in a recirculating series approach, with the first pass being crossflow, and then subsequent passes being premix emulsification (e.g., Wei et al. [79]).

Table 2. Nanosized entities produced by membranes in emulsion mode.

\begin{tabular}{|c|c|c|c|c|}
\hline $\begin{array}{l}\text { Dispersed phase composition } \\
\text { (loaded with) }\end{array}$ & ${ }^{\mathrm{T}}$ Type & $\begin{array}{l}\text { Continuous } \\
\text { medium }\end{array}$ & $\begin{array}{l}{ }^{2} \text { Dispersed } \\
\text { phase diameter } \\
(\mathrm{nm})\end{array}$ & Ref. \\
\hline Perylene in ethanol & $\mathrm{CF}$ & water & 50 & [80] \\
\hline Sulfur in CS & $\mathrm{CF}$ & ethanol \& $\mathrm{PVP}^{3}$ & $10-20$ & [81] \\
\hline${ }^{4} \mathrm{PMMP}$ in ethanol & $\mathrm{CF}$ & water & 10 & [82] \\
\hline${ }^{5}$ Gelucire $44 / 14$ or Compritol 888 & $\mathrm{CF}$ & water & 50 & [83] \\
\hline $\begin{array}{l}{ }^{6} \text { Lipoïd E80 (caffeine, } \\
\text { spironolactone) or Tween } 60 \text {, Span } \\
60 \mathrm{w} / \text { cholesterol in ethanol }\end{array}$ & $\mathrm{CF}$ & water & $\sim 80$ & [84] \\
\hline $\begin{array}{l}\text { Lipoïd E80 (beclomethasone } \\
\text { dipropionate or indomethacin) }\end{array}$ & $\mathrm{CF}$ & water & 50 & [85] \\
\hline Gelucire 44/14(vitamin E) & $\mathrm{CF}$ & ${ }^{7}$ water & 70 & [86] \\
\hline $\begin{array}{l}{ }^{8} \text { Liposome dispersionin aqueous } \\
\text { ammonium sulfate }\end{array}$ & PM & $\begin{array}{l}\text { water with } \\
\text { doxorubicin }\end{array}$ & $<100$ & [87] \\
\hline $\begin{array}{l}\text { Triglycerides, soybean oil, } \\
\text { trimyristin }\end{array}$ & PM & ${ }^{7}$ water & $\sim 100$ & [78] \\
\hline Mineral oil & $\mathrm{CF}$ & ${ }^{7}$ water & $\sim 450$ & [88] \\
\hline $\begin{array}{l}\text { Polycaprolactone (vitamin E) in } \\
\text { acetone and oils }\end{array}$ & $\mathrm{CF}$ & ${ }^{7}$ water & 165 & $\begin{array}{l}{[89,} \\
90]\end{array}$ \\
\hline $\begin{array}{l}\text { Polylactide in dichloromethane } \\
\text { and dodecane }\end{array}$ & $\mathrm{PM}$ & water \& ${ }^{9}$ alcohols & 350 & [38] \\
\hline${ }^{10} \mathrm{C} 8 \mathrm{TG} \mathrm{w} / \mathrm{PTSA}$ in $\mathrm{w} / \mathrm{o} / \mathrm{w}$ & PM & water & $\sim 330$ & [91] \\
\hline Polylactide in ethyl acetate & PM & water & $\sim 320$ & [79] \\
\hline $\begin{array}{l}\text { Phospholipids and cholesterol in } \\
\text { ethanol }\end{array}$ & $\mathrm{CF}$ & ${ }^{11}$ water & $\sim 210$ & {$[92$,} \\
\hline
\end{tabular}

${ }^{1} \mathrm{PM}$ : premix emulsification, CF: crossflow emulsification; ${ }^{2}$ The smallest mean (or mode, if mean is unavailable) diameter is reported; ${ }^{3}$ polyvinylpyrrolidone; ${ }^{4} 1$-phenyl- 3 -(p-methoxyl-styryl)-5(p-methoxyl-phenyl)-2-pyrazoline; ${ }^{5}$ Gelucire contains PEG 1500 esters, glycerides, unreacted PEG 1500, and a small amount of glycerol and Compritol 888 contains mono-, diandtriglycerides of primarily behenic acid $\left(\mathrm{C}_{22}\right) ;{ }^{6}$ Lipoid contains egg yolk lecithin with $82 \%$ phosphatidylcholine, $8 \%$ phosphatidylethanolamine, 3\% lysophosphatidylcholine, $3 \%$ sphingomyelin, and $4 \%$ nonpolar lipids (triglycerides, cholesterol, free fatty acids, and DL- $\alpha-$ tocopherol) in ethanol; Tween ${ }^{\circledR} 60$ and $\operatorname{Span}^{\circledR} 60$ are the non-ionic surfactants polyoxyethylene (20) sorbitol monostearate and sorbitol monostearate, respectively; ${ }^{7}$ various added surfactants were included; ${ }^{8}$ 1,2-Distearoyl-sn-glycero-3-phosphocholine andcholesterolliposomes created via thin film hydration; ${ }^{9}$ binary pairs with methanol, ethanol, and iso-propanol; ${ }^{10}$ Octanoic acid triacylglyceride and surfactants (decaglycerol monolaurate or hexaglyceryl condensed ricinoleate) with included 1,3,6,8- Pyrenetetrasulfonic acid tetrasodium salt, and Hank's solution in water 
phases; ${ }^{11}$ clove essential oil or Eugenol as a drug inclusion complex with cyclodextrins were dissolved in the water phase.

\subsection{Droplet formation mechanisms}

As discussed previously, there are several mechanismsoccurring in the membrane system that produces the final dispersed phase. Droplets can form both inside the membrane and at the surface of the membrane by localized shear forces, by interfacial tension effects which result in either Laplace or Rayleigh instabilities, and by steric hindrance between droplets casusing splitting[32, 88]. These effects are modulated by several process parameters including the membrane properties, such as pore size and tortuosity (really the network of interconnections), and pore size distribution and surface pore densityas well as, transmembrane pressure, and properties of the dispersed and continuous phases (such as, kinematic viscosities and interfacial tensions) and the number of passes allowed.Additionally, if a subsequent phase change of the dispersed droplets is used to affect the final particle size, such as, when condensing solid lipid nanoparticles, then lowering the temperature [86], and the subsequent heat transfer, will be an important consideration.

\subsection{Membrane properties that result in sub-100nm particles}

While some researchers used ultrafiltration (UF) membranes[78, 80, 82], the most common membranes used to produce emulsions wereMF membranes[81, 83-89, 92, 93]. These latter membranes have pores larger than $100 \mathrm{~nm}$, often significantly larger than the particles produced, thus, the size of the pore clearly does not entirely determine the size of the particle. In Hwang et al.[87], larger liposomes were broken up because of interactions with pore walls. Liposomes could split into smaller sizes because frictional forces between the liposome and the pore wall overcome the surface and elastic forces resisting deformation and rupture.

\subsection{Concentrations of NPs in membrane emulsification}

One of the challengesof membrane-based emulsions is they producea dispersed phase that is less concentrated than traditional non-membrane-emulsion processes, limiting their use to applications that do not require high concentrations. NP production, however, is often at low concentrations, with $10^{12}-10^{14}$ particles $/ \mathrm{mL}$ beingconsidered 
"relatively high"[94] in concentration. This particle concentration is 6-8 orders of magnitude less than the lowest concentration typically reported for emulsions.

Furthermore, membrane emulsion requires energy density inputs, usually defined as energy per unit volume of emulsion, several orders of magnitude lower than other emulsion processes withdispersed phase concentrations of a few percent[30]. Membranes in the emulsion configuration have the potential to increase NP concentrations in solution by severalorders-of-magnitude, an objective discussed earlier, while operating in the lowest energy density range for emulsion technologies.

\subsection{Temperature and particle size}

Temperature of the continuous and dispersed phases can affect the final particle size in sometimes unexpected ways. Charcosset et al.[86] found that operating the aqueous continuous phase at (or slightly below) the melting point of the dispersed phase, Gelucire 44/14, halved the size of the particles compared to operating the continuous phase at just above its melting point. Carefully choosing phase temperatures, often a parameter assumed to have little affect on size, may facilitate the production of sub$100 \mathrm{~nm}$ particles in other emulsions, even though, the specific physico-chemical mechanisms underlying such effects are unclear.

\subsection{NP chemistry}

Both inorganic and organic NPs have been fabricated using a membrane emulsion approach.Most of the cited emulsions featured an organicdispersed phase, however, some work has produced inorganic NPs in water. Chen et al. has produced sulfur NPs by pushing a solution of $\mathrm{S}$ and $\mathrm{CS}_{2}$ through anMF membrane into an ethanol non-solvent to precipitate out particles $10-20 \mathrm{~nm}$ in size[81].

The cyclic organic compounds, perylene and pyrazoline, were fabricated asdropletssmaller than $100 \mathrm{~nm}$ in sizeby Jia et al., [80, 82].In both cases, the process of pressing the dispersed phase through a membrane was compared to the addition of the dispersed phase in a stirred reactor. For both compounds, the membrane process produced a narrowerPSD and smaller-sized particles. In the pyrazoline work, an ethanolpyrazoline solution was pressed through a UF membrane into a water phase. This was compared to a batch process, wherein pyrazoline-ethanol is injected into a non-solvent, 
water. Inthe batch process, particles formed were much larger and more irregular than in the membrane process. When the reverse occurs and the non-solvent, water, is injected into a pyrazoline-ethanol solution, 30nm particles are formed. When the same chemistry is used in the membrane system, the particles are $10 \mathrm{~nm}$ in size. In making these nanosized particles, which component is added to which is critical, but it appears for at least some chemistries, membrane-based production synthesizes smaller particles than conventional batch processes.

\subsection{Shelf life of nano-sized emulsions}

The stability of nano-sized emulsions, created in membrane-based processes, has been investigated by a few researchers [90,91]. Charcosset et al.[86] examined stability of Gelucire 44/14 particles loaded with vitamin E over 31 days. Particles stored in the dark were considerably more stable than thoseexposed tonatural daylight. In the dark, particles approximately $250 \mathrm{~nm}$ in size grew to $500 \mathrm{~nm}$ between 2 and 5 days and then remained a stable size for the duration of the month. Particles stored in light grew to micron-sized particles within a few days and continued to grow at an accelerating rate over the course of the month. This suggests that in the smallest-sized emulsions, storage conditions are critical to maintaining their size.

The same group looked at the stability of polycaprolactone, also loaded with vitamin $\mathrm{E}$, over six months at $40^{\circ} \mathrm{C}$ and $75 \%$ relative humidity[90]. Compared to the previous study $[86,95]$ these are harsher conditions. The authors found that, with optimized emulsion chemistry, particle size and encapsulation efficiencies remained constant for three months, but particles start to grow, albeit slowly, in between three to six months. The stability of a membrane-produced emulsion was compared to a nanoprecipitation method, in which one phase was added drop-wise into another stirred phase. The emulsions produced with membrane system were less stable than the nanoprecipitation emulsions. This was attributed to the membrane system producing slightly larger particles, $250 \mathrm{~nm}$, than the nanoprecipitation method, $185 \mathrm{~nm}$, and therefore, having a higher rate of dissolution in water.

Shima et al. formed a W/O/W (water-in-oil-in-water) emulsion and found the mean size and the encapsulation efficiencies remained unchanged after one week of 
storage at $40^{\circ} \mathrm{C}$. They compared this to a rotor/stator homogenizer and found emulsions produced with a membrane system were more stable[91], due to the finer emulsion achieved with the membrane system. In cases where a membrane can produce a finer emulsion, this enhances its shelf-stablility, however, in certain cases, storage conditions will have to be carefully controlled to ensure produced particles remain in the nano-sized range.

\section{NP synthesis via contactors or self-assembly}

While there are many examples of using a membrane in the dispersion or emulsion configurations to make nano-sized materials, there are far fewer in the contactor configuration (Table 3). In all contactor studies, inexpensive microporous polypropylene (PP)or PES membranes were used to manufacture NPs, in contrast to the dispersion method, where more expensive metal membranes were commonly studied. Both gasliquid[51, 96] and immiscible liquid-liquid[42, 44]phases have been proven to produce NPs in the contactor setup.

In the gas-liquid systems, $\mathrm{CO}_{2}$ was used as a reagent to produce several types of NPs. The authors note that membranes are most advantageous when the gas is expensive and/or hazardous because all of the gas is absorbed. While $\mathrm{CO}_{2}$ is a relatively inexpensive and benign gas, there are NP chemistries in the literature that require more hazardous gases, such as ammonia[55].

Table 3. Examples producing NPs via a membrane contactor mode.

\begin{tabular}{lll}
\hline \multicolumn{1}{c}{ Particle } & Particle diameter ${ }^{1}$ (nm) & \multicolumn{1}{c}{ Ref. } \\
\hline $\mathrm{BaSO}_{4}$ & 400 & {$[44]$} \\
$\mathrm{CaCO}_{3}, \mathrm{SrCO}_{3}, \mathrm{Al}(\mathrm{OH})_{3}$ & $50,40,50$ & {$[43,51,97]$} \\
$\mathrm{Pd}$ & $5-30$ & {$[50]$} \\
\hline
\end{tabular}

${ }^{1}$ The smallest mean (or mode, if mean is unavailable) diameter is reported here.

In Jia et al.[43], modeling of the production of $\mathrm{CaCO}_{3} \mathrm{NPs}$ from an aqueous solution of $\mathrm{Ca}(\mathrm{OH})_{2}$ and $\mathrm{CO}_{2}$ gas, used a double-film and surface renewal model to calculate absorption of $\mathrm{CO}_{2}$ per unit membrane area and compare it to the measured experimental results. The model represented experimental trends well; among others, an increase in liquid flow rates resulted in increased absorption in both the model and the experiment. The model, however, always predicts larger $\mathrm{CO}_{2}$ absorption per unit 
membrane area than measured experimentally. This was attributed to membrane fouling (and membrane wetting caused by fouling), a frequent challenge for the membrane community.

Fouling wasobserved by a measured increase in pressure drop across the membrane and confirmed with SEM images. They found that fouling could be minimized by lowering the concentration of reagents and by lowering the liquid flow rate. By lowering the concentration of reagents and the liquid flow rate, fewer particles are formed, minimizing fouling, but unfortunately, also lowering the productivity of the membrane synthesis approach.

$\mathrm{H}_{2}$ reduction of $\mathrm{PdCl}_{2}$ to $\mathrm{Pd}$ nanocrystals [50] illustrated the robustness of using a membrane contactor with potentially hazardous gases in a bubble-less absorption mode. A hollow fiber membrane module with polypropylene fibers (nominally $0.05 \mu \mathrm{m}$ diameter, $60 \%$ porosity, and $100 \mu \mathrm{m}$ wall thickness) was used in a recirculating batch reaction. The liquid was passed through the fiber lumen and the gas was on the shell side. Various surface stabilizers were evaluated and nanocrystals on the order 5-7 nm were observed in TEM images as $\sim 30 \mathrm{~nm}$ clusters. They confirmed the catalytic activity of the Pd nanocrystals using a styrene hydrogenation reaction.

In aliquid-liquid system, $\mathrm{BaSO}_{4}$ was prepared with $\mathrm{BaCl}_{2}$ and $\mathrm{K}_{2} \mathrm{SO}_{4}[42,44]$. $\mathrm{BaSO}_{4}$ particles have been prepared by the dispersion method mentioned previously. The authors found that using pore sizes much larger than the particle formed resulted in no fouling. In this case, fouling was primarily observed at the inlet of the lumen side where supersaturation and nucleation were predicted to be particularly high. It is speculated that fouling was caused by nucleating $\mathrm{BaSO}_{4}$ particles attaching to the pores and continuing to grow. An interesting question arises from the results reported by Kieffer et al. [42] due to the fact that both a polyethersulfone and a polypropylene hollow fiber module was studied with a two aqueous phase system. They attributed the differences in particle deposition (measured using x-ray transmission on the full modules) between the two modules to the fiber diameter only, whereas, in the case of the polypropylene membrane there is also a question as to the shape of the meniscus that may form where the two streams contact each other. 
Though, not strictly an approach for continuous production of NPs via a membrane contactor, Zhou et al., [98] recently reported the use of a membrane biofilm reactor to recover Pd NPs from a Pd-salt-containingaqueous waste stream. $\mathrm{H}_{2}$ was introduced through the membrane, as the reducing agent, and the Pd NPs accumulated on the surface of the biofilm. One might imagine future engineering adaptations of this strategy to a (semi-)continuous production strategy.

\section{Furtherobservations}

\subsection{In dispersion}

Perhaps the most glaring omission from work discussed, based on the dispersion methodology, is the lack of noble metal chemistries. These represent an important enduse class of NPs and their synthesis could certainly be adapted to membrane dispersion approaches.

As covered in Jia and Liu's earlier review[27] on liquid-liquid dispersion, membranes have also been adopted as simple "micro-mixers". For example, Wang et al., [99] formed CdS NPs ( $\sim 3-4 \mathrm{~nm}$ diameter) by injecting $\mathrm{N}_{2}$ gas through the pores of a hollow fiber membrane (polyacrylonitrile) to mix one reagent $\left(\mathrm{Na}_{2} \mathrm{~S}\right)$ with the other $\left(\mathrm{Cd}\left(\mathrm{NO}_{3}\right)_{2}\right)$ flowing through the lumen.

These ideas are also found in some published intellectual property, such as, Wang et al., [100]who described a process for creating ZnO NPs using a membrane dispersion system for reacting ammonium carbonates with zinc salts, in the presence of surfactants. The preferred membrane is a SS MF structure with nominal mean pore sizes ranging from 1-50 $\mu \mathrm{m}$. The calcined particle sizes were on the order of $10 \mathrm{~s} \mathrm{~nm}$. Additionally, Tran et al.,[101] recently describing the synthesis of core-shell quantum dots (such as, $\mathrm{CdSe} / \mathrm{ZnS}$ ) using a sequential reaction scheme and a "tubular membrane" to introduce the shell precursor into a stream containing freshly bulk-synthesized core NPs. The combined core-shell particles have diameters $<10 \mathrm{~nm}$. The "preferred" tubular membrane is ceramic (alumina or titania) with a MWCO between 20-200 g/mol. Thus, we can infer that more commercial embodiments will be forthcoming in the future. 


\subsection{In emulsions}

As noted, earlier, while there is considerable work in producing emulsion droplets several hundred $\mathrm{nm}$ in size, there are far fewer producing particles sub-100nm size (counting as NPs). To make the smallest-sized particles, small pore sizes appear necessary with a membrane that has low surface porosity. The types of nanoparticles created in this fashion would be materials that undergoe a phase change (inversion) or densification, when entering the continuous phase, versus a reaction.

\subsection{In membrane contactors}

As membrane contactors for the production of NPs are the least studied of the three configurations, there is significant opportunity for future work, including more examples ofnoble metal chemistry. Furthermore, systematically varying experimental controls could reveal more about the fundamental mechanisms which are important to synthesize NPs with narrow sizes distributions. Also, different NP shapes (e.g., rods) have not been investigated. Mixed NPs, with different core-and-shell configurations,represents another opportunity for sequential membrane contactorsas one can imagine having the first membrane produce the core, and then the second membrane functionalizes it, creating a shell, and so on, as suggested by Tran et al., [101].

As another "thought exercise", we can estimate a nominal membrane reactor's NP productivity (and economic potential) by just considering the membrane as a "psuedocatalytic" surface for production of nanospecies with a "turnover rate" governed by the interplay between the particle formation kinetics and the mass transfer (+ release) time scales. The number of "sites"/unit surface area may be estimated by the surface pore density and the membrane surface area. We can also convert this into a module productivity based on the surface area/module. This calculation is illustrated in the followingFigure 5 for a cylindrical membrane geometry with the assumption that the particles are synthesized on the inside surface area. The simple mass balance provides

$$
\frac{\mathrm{NPs}}{\text { tube } \cdot s}=4 \phi L t_{n} \frac{d_{t}}{d_{p}^{2}}
$$


with $t_{n}=$ turnover number, NPs/pore/s,$d_{t}=$ membrane inner diameter $(\sim 1 \mathrm{~mm}), L=$ length of tube $(\sim 10 \mathrm{~cm}), d_{p}=$ pore diameter $(\sim 20 \mathrm{~nm})$, and $\phi=$ surface porosity. We can then estimate a productivity while varying $t_{n}$ and $\phi$.

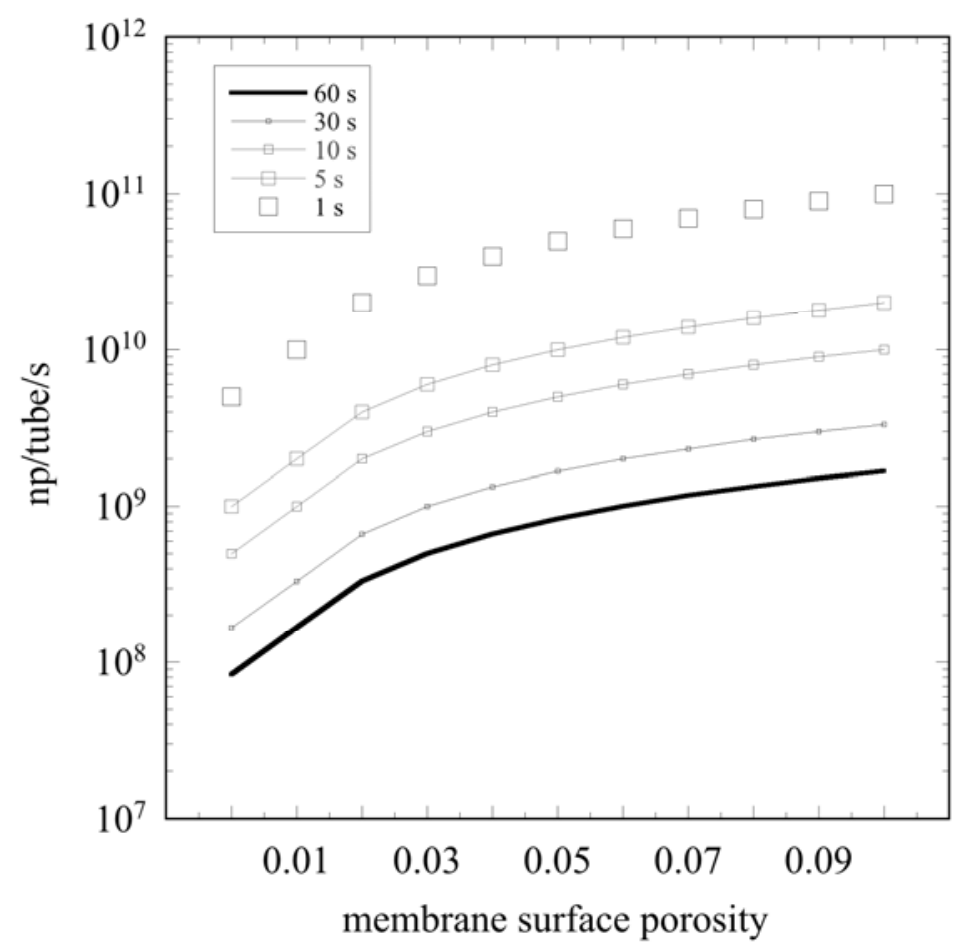

Figure 5. Hypothetical productivity of nanoparticles per membrane tube per s (NPs/tube/s) versus fractional surface porosity of the membrane. The different lines represent varying the reaction + release time (1/turnover number) from $60 \mathrm{~s}$ to $1 \mathrm{~s}$.

The overall productivity per unit volume of a reactor will scale with the membrane surface area to volume ratio when it is packaged in the module. Thus, we see that there are a number of "manufacturing" packaging variables that can be optimized for any particular turnover number. For a rough benchmark, commodity magnetite NPs in the 10-20 nm range are sold for $\sim \$ 15-20$ per $10^{15}$ particles. Larger membrane modules with $\sim 10^{4}$ fibers/tubes and 10x the length that we assumed herein can be fabricatedand would reach production rates of $\sim 10^{15}$ particles/s per module (or revenue rates per module of $\$ \sim 50,000 / \mathrm{h}$ ) if particle reaction kinetics + mass transfer timescales of $\leq 1 \mathrm{~min}$ can be achieved. 


\section{Conclusions}

Membranes have been used in a variety of configurations to produce nano-sized materials with a variety of chemistries. Even in the dispersion mode, arguably the most studied, and commercially advanced, membrane setup for producing NPs, there are many opportunities for furtheradvances. There are several process improvements that could move membrane-based processes from the bench-scale to the pilot-scale, probably most notablyusingmembrane modules made of less expensive polymeric materials instead of more costly metals and ceramics. NPsrepresentan opportunity for the membrane community to produce a high value product, perhaps better than other more traditional methods that can be limited to lower batch throughput and more costly post-processing techniques.

\section{Acknowledgement}

This work was supported within the National Science Foundation award CMMI 1435299. 


\section{References}

[1] P. Walter, E. Welcomme, P. Hallegot, N. J. Zaluzec, C. Deeb, J. Castaing, P. Veyssiere, R. Breniaux, J. L. Leveque and G. Tsoucaris, Early use of PbS nanotechnology for an ancient hair dyeing formula, Nano Lett., 6 (2006) 22152219.

[2] V. K. Varadan, A. S. Pillai, D. Mukherji, M. Dwivedi and L. Chen, Nanoscience and nanotechonology in engineering, World Scientific Publishing Co. Pte. Ltd., Singapore, 2010.

[3] B. Viswanathan, Nano Materials, Alpha Science, Oxford, UK, 2009.

[4] N. Sheibat-Othman, T. Burne, C. Charcosset and H. Fessi, Preparation of pHsensitive particles by membrane contactor, Colloids and Surfaces A:

Physicochemical and Engineering Aspects, 315 (2008) 13-22.

[5] D. V. Goia and E. Matijevic, Preparation of monodispersed metal particles, New J. Chem., 22 (1998) 1203-1215.

[6] I. Lisiecki, Size, shape, and structural control of metallic nanocrystals, J. Phys. Chem. B, 109 (2005) 12231-12244.

[7] J. Perez-Juste, I. Pastoriza-Santos, L. M. Liz-Marzan and P. Mulvaney, Gold nanorods: Synthesis, characterization and applications, Coord. Chem. Rev., 249 (2005) 1870-1901.

[8] L. D. Wang, J. J. Travis, A. S. Cavanagh, X. H. Liu, S. P. Koenig, P. Y. Huang, S. M. George and J. S. Bunch, Ultrathin oxide films by atomic layer deposition on graphene, Nano Lett., 12 (2012) 3706-3710.

[9] A. R. Esker, C. Mengel and G. Wegner, Ultrathin films of a polyelectrolyte with layered architecture, Science, 280 (1998) 892-895.

[10] B. D. Gates, Q. B. Xu, M. Stewart, D. Ryan, C. G. Willson and G. M. Whitesides, New approaches to nanofabrication: Molding, printing, and other techniques, Chem. Rev., 105 (2005) 1171-1196.

[11] R. W. Baker, Membrane Technology and Applications, John Wiley \& Sons Ltd., Chichester, England, 2004.

[12] P. W. Dunne, A. S. Munn, C. L. Starkey, T. A. Huddle and E. H. Lester, Continuous-flow hydrothermal synthesis for the production of inorganic nanomaterials, Philos. Trans. R. Soc. A-Math. Phys. Eng. Sci., 373 (2015).

[13] B. Khodashenas, R. Zadghaffari and S. D. Jafari, Process Intensification Approach for the Synthesis of Metal Nanoparticles: A Mini Review, Orient. J. Chem., 31 (2015) 249-257.

[14] L. L. Lazarus, C. T. Riche, B. C. Marin, M. Gupta, N. Malmstadt and R. L. Brutchey, Two-phase microfluidic droplet flows of ionic liquids for the synthesis of gold and silver nanoparticles, ACS Applied Materials \& Interfaces, 4 (2012) 3077-3083.

[15] B. P. Mason, K. E. Price, J. L. Steinbacher, A. R. Bogdan and D. T. McQuade, Greener approaches to organic synthesis using microreactor technology, Chem. Rev., 107 (2007) 2300-2318. 
[16] M. Thiele, A. Knauer, A. Csaki, D. Mallsch, T. Henkel, J. M. Kohler and W. Fritzsche, High-throughput synthesis of uniform silver seed particles by a continuous microfluidic synthesis platform, Chem Eng Technol, 38 (2015) 11311137.

[17] Y. X. Zhang and L. Q. Wang, Microfluidics: fabrication, droplets, bubbles and nanofluids synthesis, in L. Q. Wang, ed., Advances in Transport Phenomena 2010, 2011, pp. 171-294.

[18] C. T. Riche, E. J. Roberts, M. Gupta, R. L. Brutchey and N. Malmstadt, Flow invariant droplet formation for stable parallel microreactors, Nat. Commun., 7 (2016).

[19] A. Jahn, J. E. Reiner, W. N. Vreeland, D. L. DeVoe, L. E. Locascio and M. Gaitan, Preparation of nanoparticles by continuous-flow microfluidics, J. Nanopart. Res., 10 (2008) 925-934.

[20] C. R. Martin, Nanomaterials - a membrane-based synthetic approach, Science, 266 (1994) 1961-1966.

[21] J. C. Hulteen and C. R. Martin, A general template-based method for the preparation of nanomaterials, J. Mater. Chem., 7 (1997) 1075-1087.

[22] V. Smuleac, R. Varma, S. Sikdar and D. Bhattacharyya, Green synthesis of Fe and $\mathrm{Fe} / \mathrm{Pd}$ bimetallic nanoparticles in membranes for reductive degradation of chlorinated organics, J Membrane Sci, 379 (2011) 131-137.

[23] V. Smuleac, R. Varma, B. Baruwati, S. Sikdar and D. Bhattacharyya, Nanostructured membranes for enzyme catalysis and green synthesis of nanoparticles, Chemsuschem, 4 (2011) 1773-1777.

[24] Q. Hu and E. Marand, In situ formation of nanosized $\mathrm{TiO}_{2}$ domains within poly(amide-imide) by a sol-gel process, Polymer, 40 (1999) 4833-4843.

[25] K. K. Sirkar, Membranes, phase interfaces, and separations: Novel techniques and membranes - An overview, Ind Eng Chem Res, 47 (2008) 5250-5266.

[26] Z. Q. Jia, Progress in membrane gas-liquid reactors, J. Chem. Technol. Biotechnol., 88 (2013) 340-345.

[27] Z. Q. Jia and Z. Z. Liu, Membrane-dispersion reactor in homogeneous liquid process, J. Chem. Technol. Biotechnol., 88 (2013) 163-168.

[28] S. J. Peng and R. A. Williams, Controlled production of emulsions using a crossflow membrane, Part. Part. Syst. Charact., 15 (1998) 21-25.

[29] S. M. Joscelyne and G. Tragardh, Membrane emulsification - A literature review, J Membrane Sci, 169 (2000) 107-117.

[30] A. J. Gijsbertsen-Abrahamse, A. van der Padt and R. M. Boom, Status of crossflow membrane emulsification and outlook for industrial application, J Membrane Sci, 230 (2004) 149-159.

[31] C. Charcosset, I. Limayem and H. Fessi, The membrane emulsification process - a review, J. Chem. Technol. Biotechnol., 79 (2004) 209-218.

[32] A. Nazir, K. Schroen and R. Boom, Premix emulsification: A review, J Membrane Sci, 362 (2010) 1-11. 
[33] R. M. Boom and C. Schroen, Emulsification with micro-structured membranes and micro-engineered systems, in R. Miller and L. Liggieri, eds., Bubble and Drop Interfaces, 2011, pp. 481-502.

[34] F. Spyropoulos, D. M. Lloyd, R. D. Hancocks and A. K. Pawlik, Advances in membrane emulsification. Part A: Recent developments in processing aspects and microstructural design approaches, J. Sci. Food Agric., 94 (2014) 613-627.

[35] E. Chabanon, D. Mangin and C. Charcosset, Membranes and crystallization processes: State of the art and prospects, J Membrane Sci, 509 (2016) 57-67.

[36] S. J. Peng and R. A. Williams, Controlled production of emulsions using a crossflow membrane Part 1: droplet formation from a single pore, Chem Eng Res Des, 76 (1998) 894-901.

[37] J. D. H. Kelder, J. J. M. Janssen and R. M. Boom, Membrane emulsification with vibrating membranes: A numerical study, J Membrane Sci, 304 (2007) 50-59.

[38] H. Sawalha, Y. Fan, K. Schroen and R. Boom, Preparation of hollow polylactide microcapsules through premix membrane emulsification: effects of nonsolvent properties, J Membrane Sci, 325 (2008) 665-671.

[39] N. A. Wagdare, A. T. M. Marcelis, O. B. Ho, R. M. Boom and C. J. M. van Rijn, High throughput vegetable oil-in-water emulsification with a high porosity microengineered membrane, J Membrane Sci, 347 (2010) 1-7.

[40] Y. C. Lu, T. B. Zhang, Y. Liu and G. S. Luo, Preparation of $\mathrm{FePO}_{4}$ nano-particles by coupling fast precipitation in membrane dispersion microcontactor and hydrothermal treatment, Chem. Eng. J., 210 (2012) 18-25.

[41] G. T. Vladisavljevic, I. Kobayashi and M. Nakajima, Production of uniform droplets using membrane, microchannel and microfluidic emulsification devices, Microfluid. Nanofluid., 13 (2012) 151-178.

[42] R. Kieffer, D. Mangin, F. Puel and C. Charcosset, Precipitation of barium sulphate in a hollow fiber membrane contactor, Part I: Investigation of particulate fouling, Chem. Eng. Sci., 64 (2009) 1759-1767.

[43] Z. Jia, Q. Chang, J. Qin and H. Sun, Preparation of nanoparticles with a continuous gas/liquid membrane contactor: absorption process, J Membrane Sci, 352 (2010) 50-54.

[44] R. Kieffer, D. Mangin, F. Puel and C. Charcosset, Precipitation of barium sulphate in a hollow fiber membrane contactor: Part II The influence of process parameters, Chem. Eng. Sci., 64 (2009) 1885-1891.

[45] P. Guo, C. R. Martin, Y. P. Zhao, J. Ge and R. N. Zare, General method for producing organic nanoparticles using nanoporous membranes, Nano Lett., 10 (2010) 2202-2206.

[46] I. Kobayashi, M. Yasuno, S. Iwamoto, A. Shono, K. Satoh and M. Nakajima, Microscopic observation of emulsion droplet formation from a polycarbonate membrane, Colloid Surf. A-Physicochem. Eng. Asp., 207 (2002) 185-196.

[47] N. A. Wagdare, A. T. M. Marcelis, R. M. Boom and C. J. M. van Rijn, Porous microcapsule formation with microsieve emulsification, J. Colloid Interface Sci., 355 (2011) 453-457. 
[48] A. J. Abrahamse, R. van Lierop, R. G. M. van der Sman, A. van der Padt and R. M. Boom, Analysis of droplet formation and interactions during cross-flow membrane emulsification, J Membrane Sci, 204 (2002) 125-137.

[49] S. W. Li, J. H. Xu, Y. J. Wang and G. S. Luo, Modeling of nano-particle precipitation process in a membrane dispersion micro-structured reactor, Powder Technol., 195 (2009) 213-220.

[50] Z. Q. Jia, H. J. Sun, Q. Y. Gu, H. Z. Lang and H. Abuduwayit, Preparation of palladium nanocrystals by membrane absorption of hydrogen, J Membrane Sci, 380 (2011) 63-67.

[51] Z. Jia, Q. Chang and A. Mamat, Preparation of nanoparticles with a semi-batch gas-liquid membrane contactor, Chemical Engineering and Processing: Process Intensification, 50 (2011) 810-814.

[52] Z. Jia, Z. Liu and F. He, Synthesis of nanosized $\mathrm{BaSO}_{4}$ and $\mathrm{CaCO}_{3}$ particles with a membrane reactor: effects of additives on particles, Jourmal of Colloid and Interface Science, 266 (2003) 322-327.

[53] Z. Jia and Z. Lui, Synthesis of nanosized $\mathrm{BaSO}_{4}$ particles with a membrane reactor: effects of operating parameters on particles, J Membrane Sci, 209 (2002) 153-161.

[54] G. Chen, G. Luo, X. Yang, Y. Sun and J. Wang, Anatase-TiO $\mathrm{T}_{2}$ nano-particle preparation with a micro-mixing technique and its photocatalytic performance, Mater. Sci. Eng., A, 380 (2004) 320-325.

[55] L. Du, J. Tan, K. Wang, Y. Lu and G. Luo, Controllable preparation of $\mathrm{SiO}_{2}$ nanoparticles using a microfiltration membrane dispersion microreactor, Ind. Eng. Chem. Res., 50 (2011) 8536-8541.

[56] G. G. Chen, G. S. Luo, L. M. Yang, J. H. Xu, Y. Sun and J. D. Wang, Synthesis and size control of $\mathrm{CaHPO}_{4}$ particles in a two-liquid phase micro-mixing process, J. Cryst. Growth, 279 (2005) 501-507.

[57] S. Hassani, A. Laouini, H. Fessi and C. Charcosset, Preparation of chitosan-TPP nanoparticles using microengineered membranes- effect of parameters and encapsulation of tacrine, Colloids and Surfaces A: Physicochemical and Engineering Aspects, 482 (2015) 34-43.

[58] L. Du, Y. J. Wang, Y. C. Lu and G. S. Luo, Process intensification of BaSO4 nanoparticle preparation with agitation of microbubbles, Powder Technol., 247 (2013) 60-68.

[59] C. Huang, Y. Wang and G. Luo, Preparation of highly dispersed and small-sized $\mathrm{ZnO}$ nanoparticles in a membrane dispersion microreactor and their photocatalytic degradation, Industrial and Engineering Research, 52 (2013) 56835690 .

[60] S. Li, J. Xu and G. Luo, Control of crystal morphology through supersaturation ratio and mixing conditions, J. Cryst. Growth, 304 (2007) 219-224.

[61] G. G. Chen, G. S. Luo, J. H. Xu and J. D. Wang, Membrane dispersion precipitation method to prepare nanoparticles, Powder Technol., 139 (2004) 180185. 
[62] Y. Lu, Y. Liu, C. Zhou and G. Luo, Preparation of $\mathrm{Li}_{2} \mathrm{CO}_{3}$ nanoparticles by carbonation reaction using a microfiltration membrane dispersion microreactor, Ind. Eng. Chem., 53 (2014) 11015-11020.

[63] Y. Wang, D. Xu, H. Sun and G. Luo, Preparation of pseudoboehmite with a large pore volume and a large pore size by using a membrane-dispersion microstructured reactor through the reaction of $\mathrm{CO} 2$ and $\mathrm{NaAlO}_{2}$ solution, Ind. Eng. Chem., 50 (2011) 3889-3894.

[64] T. Zhang, Y. Wang, G. Luo and S. Bai, Preparation of highly dispersed precipitated nanosilica in a membrane dispersion microreactor, Chem. Eng. J., 258 (2014) 327-333.

[65] Y. Wang, C. Zhang, S. Bi and G. Luo, Preparation of ZnO nanoparticles using the direct precipitation method in a membrane dispersion micro-structured reactor, Powder Technol., 202 (2010) 130-136.

[66] Q. A. Wang, J. X. Wang, M. Li, L. Shao, J. F. Chen, L. Gu and Y. T. An, Largescale preparation of barium sulphate nanoparticles in a high-throughput tube-intube microchannel reactor, Chem. Eng. J., 149 (2009) 473-478.

[67] K. Wang, Y. J. Wang, G. G. Chen, G. S. Luo and J. D. Wang, Enhancement of mixing and mass transfer performance with a microstructure minireactor for controllable preparation of $\mathrm{CaCO} 3$ nanoparticles, Ind. Eng. Chem. Res., 46 (2007) 6092-6098.

[68] J. Jiang, J. Liu, C. Liu, G. Zhang, X. Gong and J. Liu, Roles of oleic acid during micropore dispersing preparation of nano-calcium carbonate particles, Appl. Surf. Sci., 257 (2011) 7047-7053.

[69] L. Du, Y. Wang and G. Luo, In situ preparation of hydrophobic $\mathrm{CaCO}_{3}$ nanoparticles in a gas-liquid microdispersion process, Particuology, 11 (2013) 421-427.

[70] T. Zhang, Y. Lu, J. Liu, K. Wang and G. Luo, Continuous ammonium silicofluoride ammonification for $\mathrm{SiO}_{2}$ nanoparticles preparation in a microchemical system, Ind. Eng. Chem. Res., 52 (2013) 5757-5764.

[71] Y. Lee, J. Lee, C. J. Bae, J. G. Park, H. J. Noh, J. H. Park and T. Hyeon, Largescale synthesis of uniform and crystalline magnetite nanoparticles using reverse micelles as nanoreactors under reflux conditions, Adv. Funct. Mater., 15 (2005) 503-509.

[72] G. Merga, R. Wilson, G. Lynn, B. H. Milosavljevic and D. Meisel, Redox catalysis on "naked" silver nanoparticles, J. Phys. Chem. C, 111 (2007) 1222012226.

[73] D. L. Marchisio and R. O. Fox, Solution of population balance equations using the direct quadrature method of moments, J. Aerosol Sci, 36 (2005) 43-73.

[74] J.-M. Commenge and L. Falk, Villermaux-Dushman protocol for experimental characterization of micromixers, Chemical Engineering and Processing: Process Intensification, 50 (2011) 979-990.

[75] K. Kandori, K. Kishi and T. Ishikawa, Formation mechanisms of monodispersed w/o emulsions by SPG filter emulsification method, Colloids and Surfaces, 61 (1991) 269-279. 
[76] R. Lostritto, R. T. Lostritto, L. Goei and S. L. Silverstri, Theoretical considerations of drug release from submicron oil-in-water emulsions, Journal of Parenteral Science and Technology, 41 (1987) 214-219.

[77] G. T. Vladisavljevic, U. Lambrich, M. Nakajima and H. Schubert, Production of o/w emulsions using SPG membranes, ceramic alpha-aluminium oxide membranes, microfluidizer and a silicon microchannel plate - a comparative study, Colloids and Surfaces A: Physicochemical and Engineering Aspects, 232 (2004) 199-207.

[78] S. Joseph and H. Bunjes, Preparation of nanoemulsions and solid lipid nanoparticles by premix membrane emulsification, J. Pharm. Sci., 101 (2012) 2479-2489.

[79] Q. Wei, W. Wei, B. Lai, L.-Y. Wang, Y.-X. Wang, Z.-G. Su and G.-H. Ma, Uniform-sized PLA nanoparticles: preparation by premix membrane emulsification, Int. J. Pharm., 359 (2008) 294-297.

[80] Z. Jia, D. Xiao, W. Yang, Y. Ma, J. Yao and Z. Liu, Preparation of perylene nanoparticles with a membrane mixer, J Membrane Sci, 241 (2004) 387-392.

[81] H. Chen, W. Dong, J. Ge, C. Wang and X. Wu, Ultrafine sulfur nanoparticles in conducting polymer shell as cathode materials for high performance lithium/sulfur batteries, Sci Rep, 3 (2013) 1910-1910.

[82] Z. Jia, Y. Ma, W. Yang, Z. Zhou, J. Yao and Z. Liu, Preparation of pyrazoline nanoparticles with a membrane mixer, Colloids and Surfaces A: Physicochemical and Engineering Aspects, 276 (2006) 22-27.

[83] C. D'Oria, C. Charcosset, A. A. Barresi and H. Fessi, Preparation of solid lipid particles by membrane emulsification-influence of process parameters, Colloids and Surfaces A: Physicochemical and Engineering Aspects, 338 (2009) 114-118.

[84] T. T. Pham, C. Jaafar-Maalej, C. Charcosset and H. Fessi, Liposome and niosome preparation using a membrane contactor for scale-up, Colloids and Surfaces B: Biointerfaces, 94 (2012) 15-21.

[85] C. Jaafar-Maalej, C. Charcosset and H. Fessi, A new method for liposome preparation using a membrane contactor, Journal of liposome research, 21 (2011) 213-220.

[86] C. Charcosset, A. El-Harati and H. Fessi, Preparation of solid lipid nanoparticles using a membrane contactor, J. Controlled Release, 108 (2005) 112-120.

[87] T. Hwang, T. J. Park, W. G. Koh, I. W. Cheong, S. W. Choi and J. H. Kim, Fabrication of nano-scale liposomes containing doxorubicin using Shirasu porous glass membrane, Colloids and Surfaces A: Physicochemical and Engineering Aspects, 392 (2011) 250-255.

[88] R. A. Williams, S. J. Peng, D. A. Wheeler, N. C. Morley, D. Taylor, M. Whalley and D. W. Houldsworth, Controlled production of emulsions using a crossflow membrane: Part 2: industrial scale manufacture, Chem. Eng. Res. Des., 76 (1998) 902-910.

[89] N. Khayata, W. Abdelwahed, M. F. Chehna, C. Charcosset and H. Fessi, Preparation of vitamin E loaded nanocapsules by the nanoprecipitation method: 
from laboratory scale to large scale using a membrane contactor, Int. J. Pharm., 423 (2012) 419-427.

[90] N. Khayata, W. Abdelwahed, M. F. Chehna, C. Charcosset and H. Fessi, Stability study and lyophilization of vitamin E-loaded nanocapsules prepared by membrane contactor, Int. J. Pharm., 439 (2012) 254-259.

[91] M. Shima, Y. Kobayashi, T. Fujii, M. Tanaka, Y. Kimura, S. Adachi and R. Matsuno, Preparation of fine $\mathrm{W} / \mathrm{O} / \mathrm{W}$ emulsion through membrane filtration of coarse $\mathrm{W} / \mathrm{O} / \mathrm{W}$ emulsion and disappearance of the inclusion of outer phase solution, Food Hydrocolloids, 18 (2004) 61-70.

[92] C. Sebaaly, C. Charcosset, S. Stainmesse, H. Fessi and H. Greige-Gerges, Clove essential oil-in-cyclodextrin-in-liposomes in the aqueous and lyophilized states: From laboratory to large scale using a membrane contactor, Carbohydr. Polym., 138 (2016) 75-85.

[93] C. Sebaaly, H. Greige-Gerges, G. Agusti, H. Fessi and C. Charcosset, Large-scale preparation of clove essential oil and eugenol-loaded liposomes using a membrane contactor and a pilot plant, Journal of liposome research, 26 (2016) 126-138.

[94] D. D. Evanoff and G. Chumanov, Size-controlled synthesis of nanoparticles. 1. "silver-only" aqueous suspensions via hydrogen reduction, J. Phys. Chem. B, 108 (2004) 13948-13956.

[95] C. Charcosset and H. Fessi, A new process for drug loaded nanocapsules preparation using a membrane contactor, Drug Dev. Ind. Pharm., 31 (2005) 987992.

[96] Z. Jia, Q. Chang, J. Qin and A. Mamat, Preparation of calcium carbonate nanoparticles with a continuous gas-liquid membrane contactor: particles morphology and membrane fouling, Chin. J. Chem. Eng., 21 (2013) 121-126.

[97] Z. Jia, Q. Chang, J. Qin and X. Hong, Preparation of nanoparticles with a gasliquid membrane contactor, J Membrane Sci, 342 (2009) 1-5.

[98] C. Zhou, A. Ontiveros-Valencia, Z. Wang, J. Maldonado, H.-P. Zhao, R. Krajmalnik-Brown and B. E. Rittmann, Palladium recovery in a $\mathrm{H}_{2}$-based membrane biofilm reactor: formation of $\operatorname{Pd}(0)$ nanoparticles through enzymatic and autocatalytic reductions, Environmental Science \& Technology, 50 (2016) 2546-2555.

[99] H. Z. Wang, J. Wei, M. H. Yu, Y. G. Li, J. F. Sun and M. F. Zhu, Hollow fiber membrane as a low-cost and high-efficient micromixer to prepare $\mathrm{CdS}$ nanoparticles, Chem. Lett., 35 (2006) 1156-1157.

[100] Y. Wang, G. Luo, J. Wang, T. Huang, R. Zu, K. Wang, Q. Huangkai and L. Liu, Nanometer zinc oxide particle preparation method, China CN103553116 A 2014.

[101] V. H. Tran, S. J. Niehaus, H. Weller and D. Ness, Method for producing core/shell nanoparticles and core/shell nanoparticles, Germany US20150214433 2015. 

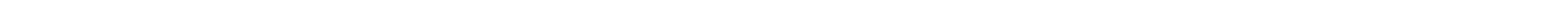